\title{
Jean-Michel Sedaine, Maillard, ou Paris sauvé et Raimond V, comte de Toulouse
}

\section{Paola Martinuzzi}

\section{(2) OpenEdition}

1 Journals

\section{Edizione digitale}

URL: http://journals.openedition.org/studifrancesi/5343

DOI: $10.4000 /$ studifrancesi.5343

ISSN: 2421-5856

\section{Editore}

Rosenberg \& Sellier

\section{Edizione cartacea}

Data di pubblicazione: 1 dicembre 2016

Paginazione: 531

ISSN: 0039-2944

\section{Notizia bibliografica digitale}

Paola Martinuzzi, « Jean-Michel Sedaine, Maillard, ou Paris sauvé et Raimond V, comte de Toulouse »,

Studi Francesi [Online], 180 (LX | III) | 2016, online dal 01 janvier 2017, consultato il 17 septembre 2020. URL : http://journals.openedition.org/studifrancesi/5343; DOI : https://doi.org/10.4000/studifrancesi. 5343

Questo documento è stato generato automaticamente il 17 settembre 2020.

\section{(c)}

Studi Francesi è distribuita con Licenza Creative Commons Attribuzione - Non commerciale - Non opere derivate 4.0 Internazionale. 


\title{
Jean-Michel Sedaine, Maillard, ou Paris sauvé et Raimond V, comte de Toulouse
}

\author{
Paola Martinuzzi
}

\section{NOTIZIA}

JEAN-MICHEL SEDAINE, Maillard, ou Paris sauvé et Raimond V, comte de Toulouse, édition présentée, établie et annotée par John Dunkley, Cambridge, The Modern Humanities Research Association, 2015, «Phoenix», 265 pp.

1 Jean-Michel Sedaine è ricordato soprattutto per l'opera in musica Le Déserteur, per la composizione di opéras-comiques e per avere dato forma scenica alle idee di Diderot nel dramma Le Philosophe sans le savoir (1765), un'opera che conobbe un successo duraturo. John Dunkley, professore emerito dell'Università di Aberdeen, presenta in questo volume l'edizione critica di due opere di Sedaine poco note: Maillard ou Paris sauvé (1770) e Raimond V (1778).

2 La tragedia Maillard, ambientata durante la Guerra dei Cent'anni, ha per protagonisti il ribelle Étienne Marcel e il suo antagonista, Jean Maillard; l'azione si svolge nel 1358, nel giorno della morte di Marcel. Il testo teatrale, che riporta nelle note le varianti manoscritte rispetto all'edizione del 1788, è preceduto da una lunga introduzione che scava nelle fonti storiche per poi metterle a confronto con la scelta letteraria di Sedaine. Lo studioso giunge ad affermare che fonte principale dello scrittore sono le Chroniques di Froissart. Da Froissart deriva l'impianto drammaturgico, la scelta di identificare l'assassino di Marcel con Maillard. Ma Sedaine elabora diversamente la psicologia di Marcel; non ne fa uno spirito "puro", non ne celebra la rivolta per i principi di partecipazione democratica, ne mostra invece le ambiguità; l'accento, rileva Dunkley, non è messo sul re che agisce contro gli interessi del popolo, ma su colui che tradisce il re. Il personaggio di Maillard, celebrato e visto come «soutien d'un régime 
consacré par sa propre longévité», poteva essere letto dai contemporanei di Sedaine alla luce dell'attualità, come un riflesso di Malesherbes. I possibili compromessi con la situazione politica nel regno di Luigi XV e poi di Luigi XVI, portarono all'archiviazione di questa «tragédie nationale», rifiutata ripetutamente dalla Comédie Française e mai rappresentata.

3 Parallelamente alla vicenda politica, è sviluppato un tema sentimentale: i rispettivi figli di Marcel e di Maillard sono uniti segretamente in matrimonio, ma il loro amore è ostacolato dai padri. Secondo lo studioso, nel disegno di Sedaine prevale la valorizzazione delle virtù familiari e dell'«héroïsme patriotique» (p. 52). L'Introduzione dedica uno spazio alla ricezione di questa tragedia in prosa (forma rara per la sua epoca), da parte di Diderot, di Voltaire, di Grimm, dell'attore Le Kain.

4 Anche la commedia eroica Raimond $V$, che viene pubblicata in questo volume per la prima volta, poteva offrire una interpretazione del passato come immagine della situazione politica presente. Secondo Dunkley, essa funge da replica complementare a Maillard. L'argomento della commedia, che fu rappresentata due volte alla Comédie Française, ruota intorno al ruolo non solo artistico ma anche politico che i trovatori ebbero nella vita di corte e alla repressione dell'eresia degli albigesi da parte di Raimondo V, conte di Tolosa, nel XII secolo. Nell'introduzione, l'attenzione del lettore viene portata sulle vicende della censura che riguardarono questa commedia e sull'analogia evidente fra l'ingerenza delle istituzioni nella vita teatrale del medioevo (come espone la pièce) e durante l'ancien régime. 